\title{
Audiovisual presentation demonstrates that selective adaptation in speech perception is purely auditory
}

\author{
MARTIN ROBERTS and QUENTIN SUMMERFIELD \\ MRC Institute of Hearing Research, University of Nottingham, Nottingham NG7 2RD, England
}

\begin{abstract}
Both auditory and phonetic processes have been implicated by previous results from selective adaptation experiments using speech stimuli. It has proved difficult to dissociate their individual contributions because the auditory and phonetic structure of conventional acoustical stimuli are mutually predictive. In the present experiment, the necessary dissociation was achieved by using an audiovisual adaptor consisting of an acoustical [be] synchronized to a video recording of

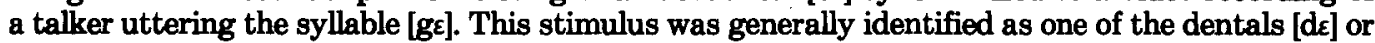
$[\partial \varepsilon]$. It produced an adaptation effect, measured with an acoustical [be-d $\varepsilon$ ] test continuum, identical in size and direction to that produced by an acoustical [be]-an adaptor sharing its acoustical structure-and opposite in direction to that produced by an acoustical [ds]-an adaptor sharing its perceived phonetic identity. Thus, the result strongly suggests that auditory rather than phonetic levels of processing are influenced in selective adaptation.
\end{abstract}

Since the introduction of the selective adaptation paradigm to research on speech perception (Eimas \& Corbit, 1973), many questions have been raised concerning the nature and loci of the processes underlying the effects obtained. The paradigm is straightforward. A series of speech sounds varying in some distinctive acoustic parameter is created, usually spanning two phonetic categories. These test syllables are randomized and presented for identification under two conditions. In the baseline condition, the individual syllables are presented for identification in isolation. In the adaptation condition, each test syllable is presented following a number of repetitions of an adapting syllable. In each condition, an ogive is fitted to the identification functions from several presentations of each syllable in a series. The point of response equiprobability, the phoneme boundary, is determined. The position of the boundary in the series usually changes following adaptation, and the size of the change provides a measure of the adaptation effect. When the adaptor is an unambiguous syllable corresponding to one or the other of the endpoints of the test continuum, the boundary usually moves toward that syllable. If, for instance, the test series embraces a phonetic contrast in voicing (e.g., [ba-pha]), following adaptation with [ba] a greater proportion of test syllables, particularly in the boundary region, are identified as [ $\mathrm{p}^{\mathrm{h}} \mathrm{a}$ ], and the phoneme boundary shifts toward the [ba] end of the series.

Originally, it was proposed (Cooper, 1974; Eimas

We thank Mark Haggard, Chris Darwin, Peter Bailey, and Peter Howell for their comments on an earlier draft of the manuscript.
\& Corbit, 1973) that these effects reflected the fatigue of "linguistic feature detectors," hypothetical neural units or arrays of units sensitive to the phonetic attributes of speech sounds. Other authors (e.g., Ganong, 1978; Howell, 1980; Tartter \& Eimas, 1975; Bailey, Note 1; Sawusch \& Jusczyk, Note 2) have shown that the size of adaptation effects depends upon the degree of spectral overlap between adaptor and test syllables and have counterargued that a significant component, if not all, of the effect is auditory rather than phonetic.

A wholly rigorous test of either claim would require that the acoustical structure of speech stimuli be dissociated from the phonetic percepts they engender. That is, it would be necessary to create stimuli that either possessed completely noncontiguous spectrotemporal specifications but produced the same phonetic percept or possessed identical acoustics but were perceived as belonging to different phonetic categories. The first alternative can be approached by synthesizing the same stop consonant before vowels with radically different formant center frequencies (e.g., Bailey, Note 1), or in syllables representing the outputs of vocal tracts of different lengths such that the formant peaks of one align with the valleys between the peaks in the other (Sawusch, 1977), or in syllables in which contrastive information is carried on a formant present in one syllable but absent in the other (Bailey, Note 1).

Less adaptation overall is obtained with these procedures compared with those in which adaptor and test stimuli are drawn from the same series of syllables and generally have more in common acoustically. The finding that the size of the effect is re- 
duced suggests that a significant component of the adaptation effect may be auditory. However, because the effect is not eliminated, a further component remains to be explained. That component could result from the operation of processes such as response biases (Diehl, Elman, \& McCusker, 1978; Diehl, Lang, \& Parker, 1980), or holistic syllable adaptation (Darwin, 1976), or, indeed, adaptation at a phonetic level of analysis. Alternatively, it may be attributable to the failure to completely eliminate spectrotemporal contiguity between adaptors and test syllables.

The alternative strategy for achieving the dissociation -that of creating stimuli with identical acoustical specifications that are nonetheless identified in different phonetic categories-has also been explored. Sawusch and Pisoni (1976), for instance, used a phonetically ambiguous stimulus extracted from near the middle of a [b-d] test series as an adaptor. Different groups of subjects were biased by instructions to identify this adaptor in different phonetic classes. Both groups evinced only small and nonsignificant amounts of adaptation, suggesting that the biasing instructions had little or no effect. A post hoc division of the subjects according to whether the adaptor fell on the [b] or [d] side of their individual phoneme boundaries showed small but systematic effects with boundaries moving toward the adaptor. Sawusch and Pisoni did not report how their subjects actually identified each adaptor, so the basis of their effectacoustic or phonetic-remains subject to inference from the average behavior in the baseline condition.

Other attempts to dissociate acoustics from phonetics (e.g., Blumstein, Stevens, \& Nigro, 1977; Ganong, Note 3) have been accomplished by synthesizing syllables whose acoustic configurations deviated substantially from their natural representations. Blumstein et al., for example, tested subjects on a [ba-da-ga] continuum following adaptation with a "conflicting cue" stimulus. This stimulus combined dental formant-frequency transitions with a velar burst (i.e., a 10-msec patch of noise centered on $1,640 \mathrm{~Hz}$ ). Five of eight subjects identified this stimulus as [ga], yet reported fewer [da] identifications after adaptation; that is, their [da-ga] boundaries moved toward the center of the [da] category. From this and other results, Blumstein et al. concluded that the adaptation effect was "based upon the acoustic information in the signal rather than the phonetic label given the stimulus by the subject" (p. 1310). However, the remaining three subjects identified this same conflicting cue adaptor as [da], suggesting that the acoustic/phonetic dissociation in this instance was not perfect. The outcome may be explained by the proximity of the [g] burst to the onset frequency of the second formant in a stimulus that, without such a burst, was unanimously identified as [da].
In another attempt to dissociate the acoustical and phonetic structure of an adaptor, Ganong (Note 3) synthesized a [sae] adaptor consisting of a fricative noise and a steady-state vowel. Despite lacking formant transitions, it reliably [d]-adapted subjects' [bae]-[dae] boundaries, assumed to be based on formant transition values. This result does not allow the dismissal of phonetic adaptation, since the consonants in the adaptor and test stimuli shared the dental place of articulation. Equally, an argument for this being an acoustic effect could be based on the fricative noise having influenced auditory detectors not necessarily restricted to the processing of formant transitions but sensitive to integrated spectral patterns of acoustic information (Ganong, 1978; Stevens \& Blumstein, 1978).

While the entire set of phenomena reviewed could reasonably be interpreted as suggesting that some auditory adaptation exists, ruling out other levels is certainly not justified. It may not be possible to isolate absolutely the acoustic and phonetic components of the adaptation process using purely acoustical stimuli, especially with regard to the dimension of place of articulation. However, by exploiting recent discoveries in audiovisual speech perception (McGurk \& MacDonald, 1976), the necessary dissociation can be achieved simply and elegantly. McGurk and MacDonald presented observers with stimuli constructed by cross-splicing audio recordings of a talker uttering one consonant-vowel (CV) syllable with video recordings of the same talker uttering a different CV syllable. The majority of observers did not perceive the conflict in these intrinsically conflicting stimuli. Rather, they perceived syllables, which, with some audiovisual combinations, corresponded to the syllable presented in one or another individual modality and which, with other combinations, corresponded to the syllable presented in neither individual modality. The combination of audio [ba] with video [ga], for instance, yielded the unambiguous percept [da] for $98 \%$ of the adult observers tested (McGurk \& MacDonald, 1976), although in a subsequent experiment (MacDonald \& McGurk, 1978) the effect occurred for only $64 \%$ of adult subjects. Nevertheless, with this technique, an utterance, although specified quite unambiguously in acoustical terms, can be modified perceptually for the majority of observers without changing its acoustical structure. In the present experiment, a stimulus was constructed by synchronizing an acoustical [be] with an optical [ge]. In accordance with McGurk and MacDonald's results, this audiovisual stimulus should generally be perceived as [de]. It was used as an adaptor with a test series whose members varied in place of articu-

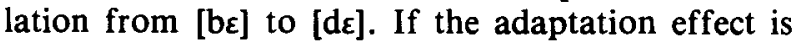
predominantly acoustical, following adaptation a greater proportion of test syllables should be identified as $[d \varepsilon]$, with the phoneme boundary on the test 
series shifting toward [be]. Alternatively, if the effect is predominantly phonetic, the outcome should be reversed, with the boundary shifting toward [de].

In addition to achieving a clear-cut dissociation of auditory and phonetic bases for adaptation, the design of this experiment provided the opportunity of gathering data relevant to an unpublished finding cited by Cooper (1975). Cooper mentioned that phoneme boundaries on a synthetic [ba-pha] continuum shifted, although not systematically, after subjects had monitored graphically presented words containing either voiced or voiceless stop consonants (e.g., "bob" and "bog" or "pop" and "pot"). Similarly, Cooper and Provezano (cited in Cooper, 1975) found shifts, although, again, not systematic ones, in subjects' phoneme boundaries after they had read silently to themselves a prose passage containing a high proportion of voiceless stops. One condition of the present experiment required subjects to watch a videotape recording of a talker repeating one of the syllables $[\mathrm{b} \varepsilon]$ or $[\mathrm{d} \varepsilon]$, with no acoustical accompaniment. This presentation was used as an adapting sequence to determine whether a purely optical specification of articulation would produce a change in subjects' subsequent identification. Although the vocalic portion of these visually presented $\mathrm{CV}$ syllables was ambiguous, the phonetic contrast between bilabial and nonbilabial articulation was precisely specified. This contrast has been shown to be perceptually potent in audiovisual speech perception (MacDonald \& McGurk, 1978; McGurk \& MacDonald, 1976; Summerfield, 1979). If perceptual adaptation can occur at the presumably central level at which optics and acoustics are combined, then subjects' identifications of acoustically presented test syllables might be affected by these purely optical adaptors.

\section{METHOD}

\begin{abstract}
Subjects
Twelve volunteers from the students and staff of the University of Nottingham served as subjects. They ranged in age from 18 to 65 years. None reported any hearing impairment, and all were native speakers of British English. All but one had had no previous experience of listening to synthetic speech. They each served in five separate 1-h sessions and were paid $£ 7.00$ for their services.
\end{abstract}

\footnotetext{
Stimuli

A nine-member [bz-dz] test series was created with an Ove $111 \mathrm{~b}$ serial resonance speech synthesizer. Each syllable was $300 \mathrm{msec}$ in duration, with $48-\mathrm{msec}$ initial formant transitions, followed by a 252-msec steady-state vowel. The onset frequencies of the second (F2) and third (F3) formants ranged in equal steps from 1,300 to $2,100 \mathrm{~Hz}(\mathrm{~F} 2)$ and from 2,400 to $3,200 \mathrm{~Hz}(\mathrm{~F} 3)$. The onset frequency of the first formant (F1) was fixed at $300 \mathrm{~Hz}$. The steadystate frequencies of these three formants were 750 (F1), 1,800 (F2), and $2,700 \mathrm{~Hz}(\mathrm{~F} 3)$. F4 and F5 were fixed at 3.4 and $5 \mathrm{kHz}$, respectively. The amplitude contour of all syllables rose to its maximum over the first $40 \mathrm{msec}$ and fell $10 \mathrm{~dB}$ over the final $100 \mathrm{msec}$ of the syllable. The fundamental frequency contour was modeled on that in a natural production of [ba] and fell quasi-linearly from 150 to $97 \mathrm{~Hz}$ over the duration of each syllable.
}

All testing was done with purely acoustical stimuli; however, there was an optical component in five of the seven adapting stimuli. The two purely acoustical adaptors were the $[\mathrm{b} \varepsilon]$ and $[\mathrm{d} \varepsilon]$ endpoint syllables from the test continuum, used in Conditions $\mathbf{A b}$ and Ad, respectively. Two adaptors were purely optical. These were videorecordings of one of the authors (M.R., who also ran the experiment) miming one of the utterances $[\mathrm{b} \varepsilon]$ and $[\mathrm{d} \varepsilon]$ and were used in Conditions $\mathrm{Vb}$ and $\mathrm{Vd}$, respectively. Three were audiovisual adaptors. Each was created by synchronizing a video recording of the same talker uttering a CV syllable with either the

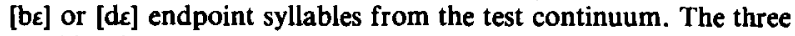
combinations were: acoustical $[b \varepsilon]$ with optical $[b \varepsilon]$ in Condition

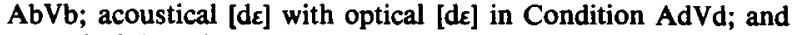
acoustical [be] with optical [ge] in Condition AbVg.

Four monochrome videotapes were recorded on a Sony U-Matic video-cassette recorder, with a flat audio frequency response from $50 \mathrm{~Hz}$ to $12 \mathrm{kHz}$. The first involved no video and was simply an audio recording of a randomization in which each test syllable occurred 12 times, with a 3-sec intertrial interval. This tape would be used to establish baseline phoneme boundaries prior to adaptation. The remaining three tapes included audiovisual stimuli and would be used in the adaptation conditions. Their formats were similar. Each included 108 trials. Each trial consisted of 50 repetitions of an audiovisual adapting stimulus with an interstimulus interval of $500 \mathrm{msec}$. This sequence was followed after a 2-sec pause by three purely acoustical test syllables drawn randomly from the test series, with an interstimulus interval of $1.5 \mathrm{sec}$. The sequence was divided into three blocks of 36 trials, with an additional 30-sec pause between blocks. The 1st, the 37th, and the 73rd trials included an additional 50 repetitions of the adapting stimulus.

The 108 trials were recorded in the following way. The speaker sat facing the video camera and his responses were recorded with the video frame extending from his shoulders to the top of his head. Two warning tones preceded each sequence of adapting sounds, which were recorded on the first audio channel of the video-cassette recorder. The speaker mouthed a syllable in synchrony with each acoustical adaptor. Synchrony of onsets to within $\pm 10 \mathrm{msec}$ was achieved in this way (see Summerfield, 1979). Immediately after the last syllable in each adapting sequence, the lens of the video camera was covered, and the three test syllables were recorded on the second audio channel of the video recorder.

On the first tape, both the acoustical and optical components of the adaptor were $[b \varepsilon]$. This tape would be used in Conditions $\mathrm{Ab}$ (in which no video would be presented), $\mathrm{Vb}$ (in which the adaptors would be presented optically and the test syllables acoustically), and $\mathrm{AbVb}$ (in which complete audiovisual adaptors and acoustical test syllables would be presented).

On the second tape, both the acoustical and optical components of the adaptor were [d $\varepsilon$ ]. This tape would be used, analogously to the first, in Conditions Ad, Vd, and AdVd. On the third tape, the acoustical component of the adaptor was [bc], while the optical component was [ge]. This tape would be used only in Condition $\mathrm{AbVg}$.

\section{Procedure}

Each subject took part in five sessions. Each session involved a practice sequence, a baseline sequence, and a test sequence. Subjects were tested individually. They sat at a small table in a sound-attenuating chamber. In front of them was a TV monitor, located at eye level. A single audiomaster loudspeaker was placed $1.00 \mathrm{~m}$ below the monitor. The leading edge of the subject's seat was positioned $1.30 \mathrm{~m}$ from the loudspeaker and $1.4 \mathrm{~m}$ from the base of the TV monitor. This configuration had previously been found to be the most likely of several arrangements considered to produce the "McGurk effect" reliably. Stimuli were presented from a Sony U-Matic video recorder. The intensity of the acoustical components of the stimuli was $45 \mathrm{~dB}$ SPL, measured $1 \mathrm{~m}$ above the front edge of the seat. A standard lamp was positioned forward and to the right of the subject and was shielded so as to 
illuminate the subject's response sheet without either dazzling the subject or reflecting in the screen of the TV monitor.

At the start of every session, subjects listened to a practice block of 36 stimuli, which included only Stimuli 1 through 3 and 7 through 9 of the test series. If their responses to these stimuli were consistent, the 108-trial baseline sequence was presented. Subjects were instructed to listen to each stimulus syllable and to identify its initial consonant as [b] or [d] by writing " $B$ " or " $D$ " on a response sheet. For the test conditions, subjects were instructed to attend to the video monitor throughout the duration of each sequence of adaptors and to keep their posture reasonably constant. Following the sequence of adaptors, they were to maintain their gaze at the screen until the three test syllables had been presented. They had then to record their responses and immediately return their gaze to the TV screen. Prior to each test condition, subjects were informed whether to expect optical, acoustical, or audiovisual sequences of adaptors. At the end of each session, subjects were asked how they had perceived the adaptor.

\section{RESULTS}

The procedures used to generate the McGurk effect in Condition $\mathrm{AbVg}$ were largely successful. All but one of the subjects followed the instruction always to observe the video monitor and were unaware of the illusory nature of the adaptor. Six of the 12 listeners identified the adapting consonant as one of the dentals [d] or [ $\theta$ ]. Five others reported nonlabial percepts. The final column in Table 1 lists the way in which each subject reported hearing the adaptor in this condition. Five subjects-Subjects $1,2,4,8$, and 11 -experienced verbal transformations during presentation of the adapting syllables. For all of these subjects, Table 1 reports the predominant percept. Of these five subjects, four did not report bilabials even as secondary percepts; the fifth, who claimed to have heard the adaptor predominantly as [ma], reported [dE] as a secondary percept.
Cumulative normal ogives were fitted to the baseline and adaptation identification functions provided by each subject in each session by the method of probits (Finney, 1971). The number of the hypothetical test stimulus corresponding to the $50 \%$ point on each function provided a measure of the position of the phoneme boundary. Differences between baseline and adaptation boundaries for each subject in each condition are tabulated in Table 1 . These differences provide a measure of each adaptation effect. They were examined first in two-tailed t tests. Purely optical adaptors did not produce significant effects [Vb: $\mathrm{t}(5)=.42, \mathrm{p}>.2$; $\mathrm{Vd}: \mathrm{t}(5)=.64, \mathrm{p}>.2]$. Purely acoustical adaptors, in comparison, produced large and systematic effects $[\mathrm{Ab}: \mathrm{t}(5)=15.00, \mathrm{p}<.001$; Ad: $\mathfrak{t}(5)=10.30, \mathrm{p}<.001$, as did the two audiovisual adaptors with compatible acoustical and optical components $[\mathrm{AbVb}: \mathrm{t}(11)=8.96, \mathrm{p}<.001$; AdVd: $t(11)=10.03, p<.001]$. The audiovisual adaptor created by combining an acoustical $[b \varepsilon]$ with an optical [ge] produced a significant shift in listeners' phoneme boundaries toward the [bz] end of the test continuum [AbVg: $\mathfrak{t}(11)=18.87, \mathrm{p}<.001]$. Finally, a post hoc analysis showed that the six subjects who identified the adaptor in Condition $\mathrm{AbVg}$ as a dental produced a slightly larger [b] adaptation effect than did the remaining six subjects. The effect, however, was not significant $[\mathrm{t}(10)=1.25, \mathrm{p}<1.0]$.

To determine whether the addition of compatible optical information to acoustical adaptors modified adaptation effects systematically, the data obtained in Conditions $\mathrm{Ab}, \mathrm{Ad}, \mathrm{AbVb}$, and AdVd were examined in a $2 \times 2 \times 2$ analysis of variance with the factors presentation: (acoustical vs. audiovisual), adaptor: (b vs. d), and test: (baseline vs. test). Sig-

Table 1

Adaptation Effects (in Stimulus Units) for Individual Subjects

\begin{tabular}{|c|c|c|c|c|c|c|c|c|}
\hline \multirow[b]{2}{*}{ Subject } & \multicolumn{7}{|c|}{ Condition } & \\
\hline & $\mathrm{Vb}$ & $\mathrm{Vd}$ & $\mathrm{Ab}$ & Ad & $\mathrm{AbVb}$ & AdVd & $\mathrm{AbVg}$ & \\
\hline $\begin{array}{l}1 \\
2 \\
3 \\
4 \\
5 \\
6\end{array}$ & $\begin{array}{r}.32 \\
.38 \\
-.19 \\
.92 \\
.02 \\
-.83\end{array}$ & $\begin{array}{r}-.12 \\
.39 \\
-.45 \\
-.28 \\
.42 \\
-.64\end{array}$ & & & $\begin{array}{r}1.25 \\
2.96 \\
.76 \\
1.71 \\
2.04 \\
.93\end{array}$ & $\begin{array}{l}-1.55 \\
-1.55 \\
-1.48 \\
-1.75 \\
-.09 \\
-1.80\end{array}$ & $\begin{array}{l}2.21 \\
2.20 \\
1.59 \\
1.62 \\
1.46 \\
1.59\end{array}$ & $\begin{array}{l}{\left[\gamma_{\epsilon}\right]} \\
{[\mathrm{d} \epsilon]} \\
{[\mathrm{kl} \epsilon]} \\
{[\mathrm{kl} \epsilon]} \\
{[\mathrm{kl} \epsilon]} \\
{[\mathrm{d} \epsilon]}\end{array}$ \\
\hline Mean & .10 & -.11 & & & 1.61 & -1.37 & 1.78 & \\
\hline $\begin{array}{r}7 \\
8 \\
9 \\
10 \\
11 \\
12\end{array}$ & & & $\begin{array}{l}2.42 \\
1.77 \\
2.07 \\
1.87 \\
2.64 \\
2.51\end{array}$ & $\begin{array}{l}-1.93 \\
-1.72 \\
-1.42 \\
-.97 \\
-1.95 \\
-1.42\end{array}$ & $\begin{array}{l}2.25 \\
1.99 \\
1.12 \\
1.85 \\
2.81 \\
2.16\end{array}$ & $\begin{array}{r}-1.85 \\
-1.73 \\
-2.13 \\
-1.81 \\
-1.67 \\
-.99\end{array}$ & $\begin{array}{l}1.74 \\
1.94 \\
1.60 \\
1.82 \\
2.36 \\
2.47\end{array}$ & $\begin{array}{l}{[\delta \varepsilon]} \\
{[\mathrm{ma}]} \\
{[\mathrm{fl} \epsilon]} \\
{[\mathrm{d} \epsilon]} \\
{[\mathrm{fl} \epsilon]} \\
{[\mathrm{d} \epsilon]}\end{array}$ \\
\hline Mean & & & 2.21 & -1.57 & 2.03 & -1.70 & 1.99 & \\
\hline Grand Mean & .10 & -.11 & 2.21 & -1.57 & 1.82 & -1.54 & 1.89 & \\
\hline
\end{tabular}

Note-Phonetic transcriptions of subjects' descriptions of the adaptor used in Condition AbVg are listed in the right-hand column. 
nificant effects of adaptor and test were found but not of presentation $[F(1,5)=.12, p>.1]$ or of its interaction with test $[F(1,5)=.24, p>.1]$, showing that the addition of compatible optical information did not contribute to the size of the adaptation effects.

A $3 \times 2$ analysis of variance with the factors presentation ( $\mathrm{Ab}$ vs. $\mathrm{AbVb}$ vs. $\mathrm{AbVg}$ ) and test (baseline vs. test) sought to determine whether the size of the adaptation effect produced by the incompatible audiovisual combination in Condition $\mathrm{AbVg}$ differed from those produced in the other two conditions in which acoustical: [be] was a component of the adaptor. Neither the main effect of presentation $[F(2,10)$ $=.30, \mathrm{p}>.1]$ nor the interaction between presentation and test $[F(2,10)=1.16, p>.1]$ were significant.

Overall, the results of the experiment are clear-cut. First, purely optical adaptors do not produce systematic adaptation effects on acoustical test continua; second, the addition of neither compatible nor incompatible optics to acoustical adaptors-that on their own give distinct and reliable percepts-modifies adaptation effects; third, even when the addition of incompatible optics changes the perceived phonetic identity of an adaptor, no change in the adaptation effect results. Overall, adaptation effects here relate to the acoustical, not the phonetic, relationships between adaptors and test syllables.

\section{DISCUSSION}

The results of this experiment provide convincing evidence that selective adaptation to speech, at least when adaptors and test syllables vary in place of articulation, depends for its effect upon the spectrotemporal relationship between the adaptor and test syllables. It appears not to depend, as has sometimes been suggested (e.g., Cooper, 1975; Diehl, 1975), upon the relationship between the perceived phonetic identities of adaptors and test syllables. Unequivocal identification of the adapting consonant in Condition $\mathrm{AbVg}$ as a dental ([d] or [छ]) was invariably accompanied by [b] adaptation. If the perceived phonetic identity of the adaptor had contributed in any way to the effects observed here, then we should have found a shift toward [d] in the phoneme boundary of all those subjects who identified this adaptor as [dz] and, possibly, little or no shift for those subjects who heard the adaptor as the intrinsically more ambiguous $\left[\theta_{\varepsilon}\right]$. However, every subject showed a reliable shift toward [be], and we conclude that the acoustical properties of the adapting syllable were responsible for its effect.

This interpretation would be undermined by a demonstration of the existence of two sites of phonetic processing serving the auditory and visual modalities independently. Rather than phonetic cate- gorization occurring after the confluence of continuous and essentially analog information from vision and audition, independent analyses would generate a phonetic feature description of the input to each modality. Summation rules would then determine which combination of features should determine the percept. With such an organization, audiovisual adaptors could generate independent phonetic adaptation at each site, but only the influence on the auditory channel would be detected with an acoustical test series.

MacDonald and McGurk (1978) considered the possibility of this form of perceptual organization. Many of their results conform to the hypothesis that, with audiovisual presentation, place features are derived from vision, while manner and voicing features are obtained from audition. Elsewhere (Summerfield, 1979), we have argued against this hypothesis. It implies, improbably, that a special mode of perception exists to serve audiovisual presentation, and it cannot handle several of the results obtained by McGurk, such as the one explored here. Furthermore, demonstrations have been contrived, using both audiovisual presentation (Rosen, Moore, \& Fourcin, Note 4) and tactile-visual presentation (Erber \& De Fillipo, 1978), in which manner and voicing could not be determined from either modality singly but only from the relative timing of events occurring across modalities, thus confounding the hypothesis of independent phonetic analyzers. These results require that phonetic categorization follows the integration of information in the two modalities, and hence they endorse the conclusion that audiovisual adaptors influence auditory, rather than phonetic, processes.

The results of Conditions $\mathrm{Vb}$ and $\mathrm{Vd}$, in which purely optical adaptors produced no measurable adaptation, provide further support. In contrast, Cooper (1975) mentioned that optically presented graphical stimuli that subjects read did produce adaptation effects on acoustical test continua. These effects were, however, unsystematic and asymmetric to a degree atypical of acoustically generated adaptation (Cooper, 1979). Our Conditions Vb and Vd have shown that lipreading does not tap whatever aspects of acoustical speech perception there are that might be influenced by graphical presentation. The result suggests that further searches for such effects would not be rewarding.

Finally, the absence of [d] adaptation-despite perception of the audiovisual adaptor in Condition $\mathrm{AbVg}$ of this experiment as [dz]-speaks to another issue. Diehl and his co-workers (e.g., Diehl et al., 1978; Diehl et al., 1980; Elman, 1979) have presented evidence to suggest that the speech adaptation effects typically obtained with procedures similar to that employed here do not reflect fatigue introduced into 
those sensory processes engaged by an adapting stimulus but, rather, reflect changes in subjects' decision criteria. Diehl et al. (1978) are noncommittal as to the level of processing at which a subject's decision criterion might undergo modification, in both their own paired-comparison procedure and that of conventional adaptation experiments. However, others (e.g., Sawusch \& Jusczyk, Note 2) have equated the term "decision criterion" with "phonetic decision rules." Sawusch and Jusczyk have suggested that contrast effects operate at a level of processing based on the "perceptual similarity" between the context and the test item. Here, however, we have argued that adaptation effects cannot be attributed to the perceived phonetic similarity between the adaptor and test syllables. If the proposal of Diehl et al. (1978) and Diehl et al. (1980) is accepted-that speech adaptation effects and contrast effects reflect the operation of a common process or set of processesthen the failure of a stimulus perceived as [dE] to [d]-adapt a [be]-[de] test continuum suggests that contrast effects themselves arise at a level distinct from that at which "phonetic decision rules" apply. It is necessary to conclude, therefore, either that the contrast explanation of speech adaptation is incorrect or that the assumed equivalence between decision criteria and phonetic decision rules is unjustified. In the latter case, contrast effects would have to be seen as originating at a subphonemic level of analysis, as proposed by Repp, Healy, and Crowder (1979).

\section{REFERENCE NOTES}

1. Bailey, P. J. Perceptual adaptation in speech: Some properties of detectors for acoustical cues to phonetic distinctions. Unpublished doctoral dissertation, Cambridge University, 1975.

2. Sawusch, J. R., \& Jusczyk, P. Adaptation and contrast in the perception of voicing (Research on Speech Perception Progress Report No. 5). Bloomington: Indiana University, 1979.

3. Ganong, W. F. An experiment on "phonetic adaptation" (Progress Report 116, 206-210, Research Laboratory of Electronics). Cambridge: Massachusetts Institute of Technology, 1975.

4. Rosen, S. M., Moore, B. C. J., \& Fourcin, A. J. Lipreading with fundamental frequency information. Proceedings of the Institute of Acoustics, 1979, 1A2, 5-8.

\section{REFERENCES}

Blumstein, S. E., Stevens, K. N., \& Nigro, G. N. Property detectors for bursts and transitions in speech perception. Journal of the Acoustical Society of America, 1977, 61, 1301-1313. Cooper, W. E. Adaptation of phonetic feature analyzers for place of articulation. Journal of the Acoustical Society of America, 1974, 56, 617-627.
Cooper, W. E. Selective adaptation to speech. In F. Restle, R. M. Shiffrin, J. N. Castellan, H. Lindman, \& D. B. Pisoni (Eds.), Cognitive theory (Vol. 1). Hillsdale, N.J: Erlbaum, 1975.

Cooper, W. E. Speech production and perception: Studies in selective adaptation. Norwood, N.J: Ablex, 1979.

DARwin, C. J. The perception of speech. In E. C. Carterette \& M. P. Friedman (Eds.), Handbook of perception (Vol. 7) Language and speech. London: Academic Press, 1976.

DiEHL, $R$. L. The effect of selective adaptation on the identification of speech sounds. Perception \& Psychophysics, 1975, 17, 48-52.

Diehl, R. L., Elman, J. L., \& McCusker, S. B. Contrast effects on stop consonant identification. Journal of Experimental Psychology: Human Perception and Performance, 1978, 4, 599-609.

Diehl, R. L., Lang, M., \& Parker, E. M. A further parallel between adaptation and contrast. Journal of Experimental Psychology: Human Perception and Performance, 1980, 6, 24-44.

Eimas, P. D., \& Corbit, J. D. Selective adaptation of linguistic feature detectors. Cognitive Psychology, 1973, 4, 99-109.

Elman, J. L. Perceptual origins of the phoneme boundary effect and selective adaptation to speech: A signal detection theory analysis. Journal of the Acoustical Society of America, 1979, 65, $190-207$.

ERBER, N. P., \& De Fillipo, C. L. Voice/mouth synthesis and tactual/visual perception of /pa, ba, ma/. Journal of the Acoustical Society of America, 1978, 64, 1015-1019.

Finney, D. J. Probit analysis. New York: Cambridge University Press, 1971.

Ganong, W. F. The selective adaptation of burst-cued stops. Perception \& Psychophysics, 1978, 24, 71-83.

Howell, P. Vowel-contingent feature detection. Perception \& Psychophysics, 1980, 27, 37-42.

MacDonald, J., \& McGurk, H. Visual influences on speech perception processing. Perception \& Psychophysics, 1978, 24, 253-257.

McGurk, H., \& MacDonald, J. Hearing lips and seeing voices. Nature, 1976, 264, 746-748.

Repp, B. H., Henly, A. F., \& Crowder, R. G. Categories and context in the perception of isolated steady-state vowels. Journal of Experimental Psychology: Human Perception and Performance, 1979, 5, 129-145.

SAwUsch, J. R. Peripheral and central processes in selective adaptation of place of articulation' in stop consonants. Journal of the Acoustical Society of America, 1977, 62, 738-750.

SAwusch, J. R., \& Pisoni, D. B. Response organization in selective adaptation to speech sounds. Perception \& Psychophysics, 1976, 20, 413-418.

Stevens, K. N., \& Blumstein, S. E. Invariant cues for place of articulation in stop consonants. Journal of the Acoustical Society of America, 1978, 64, 1358-1368.

SummerField, $Q$. Use of visual information for phonetic perception. Phonetica, 1979, 36, 314-331.

TARTter, V. C., \& Eimas, P. D. The role of auditory feature detectors in the perception of speech. Perception \& Psychophysics, 1975, 18, 293-298.

(Manuscript received May 11, 1981; revision accepted for publication June 8, 1981.) 